\title{
A Self-Assembled Donor Comprising Quantum Dots and Fluorescent Proteins for Long-Range Fluorescence Resonance Energy Transfer
}

\author{
Huachang Lu $u^{a}$, Oliver Schöps ${ }^{b}$, Ulrike Woggon $^{b} *$ and Christof M. Niemeyer ${ }^{a} *$
}

(a) Technische Universität Dortmund, Fakultät Chemie, Biologisch-Chemische Mikrostrukturtechnik, Otto-Hahn Str. 6, D-44227 Dortmund, Germany.

(b) Technische Universität Dortmund, Fakultät Physik, Otto-Hahn Str. 4, D-44227 Dortmund, Germany.

E-mail: christof.niemeyer@tu-dortmund.de; ulrike.woggon@tu-dortmund.de

Photobleaching of EYFP-A24 conjugates. The PL and absorbance of EYFP-A24 conjugates were compared before and after photobleaching.

(A)

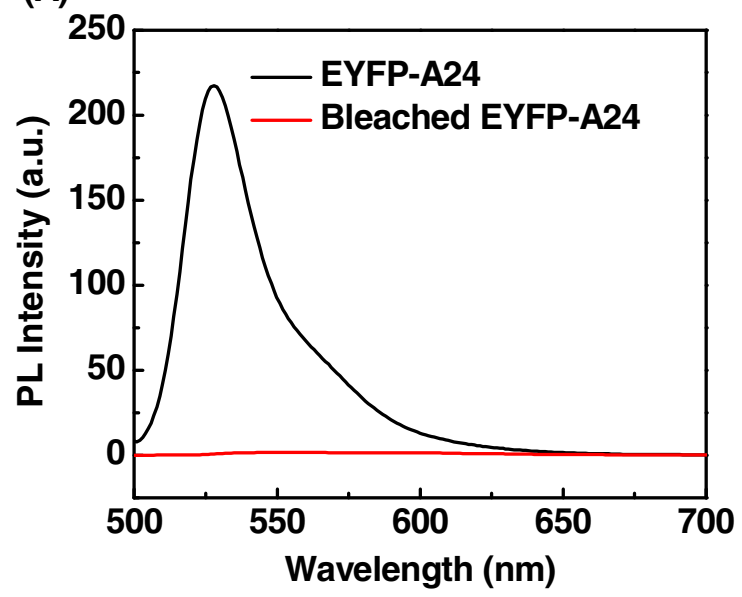

(B)

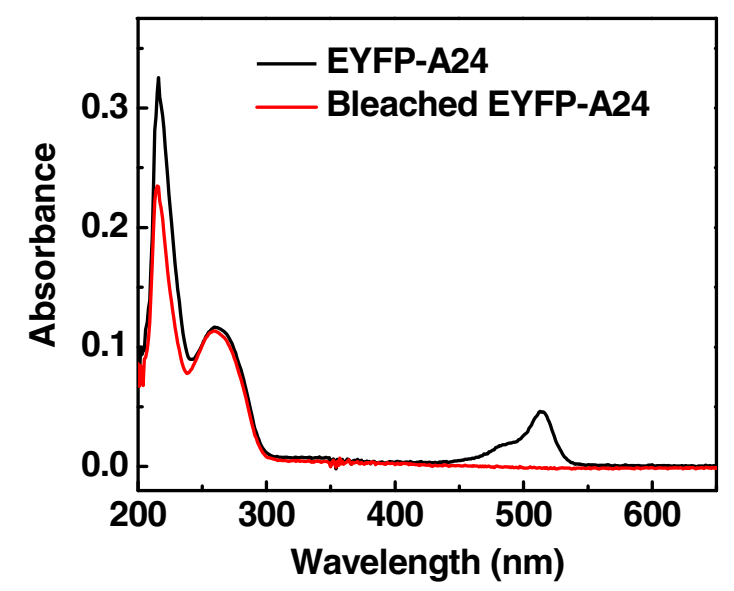


Figure S1. The samples were diluted to $0.5 \mu \mathrm{M}$ in TETBS buffer and excited at $488 \mathrm{~nm}$. (A) Fluorescent spectra comparison: the PL completely disappeared after irradiating with laser. (B) Absorbance comparison of EYFP-A24 and bleached EYFP-A24 conjugates: The UV absorption peaks below $300 \mathrm{~nm}$ remain almost unchanged, while the absorption peak of EYFP centered at $514 \mathrm{~nm}$ disappears completely after photobleaching.

PL enhancement comparison of QD/EYFP and QD/EYFP-A24 conjugates. The titration experiments of QD/EYFP and QD/EYFP-A24 indicated that the surface coverages of the QD with either EYFP or EYFP-A24 conjugates were very similar, i.e., in the range of 13-16. This suggests that the oligonucleotide A24 attached to the EYFP had only little effects on the effective size of the protein. However, PL enhancement was significantly greater in the case of QD/EYFP as compared to QD/EYFP-A24. The reasons for this observation are currently unclear. However, one may speculate that the increased polarity of the EYFP-A24 might lead to a less efficient hydrophobic shielding of the QDs surfaces, as compared to the pure EYFP.

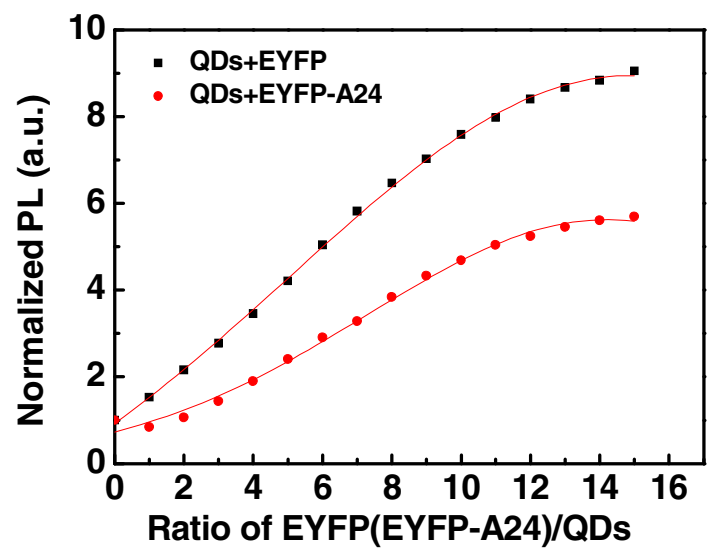

Figure S2. PL enhancement comparison of QD-EYFP (black squares) and QD-EYFP-A24 (red dots) conjugates at increasing of EYFP(EYFP-A24)/QD ratios. 
Original data of QD/EYFP-A24 PL spectra. The original data before EYFP background subtraction and normalization.

(A)

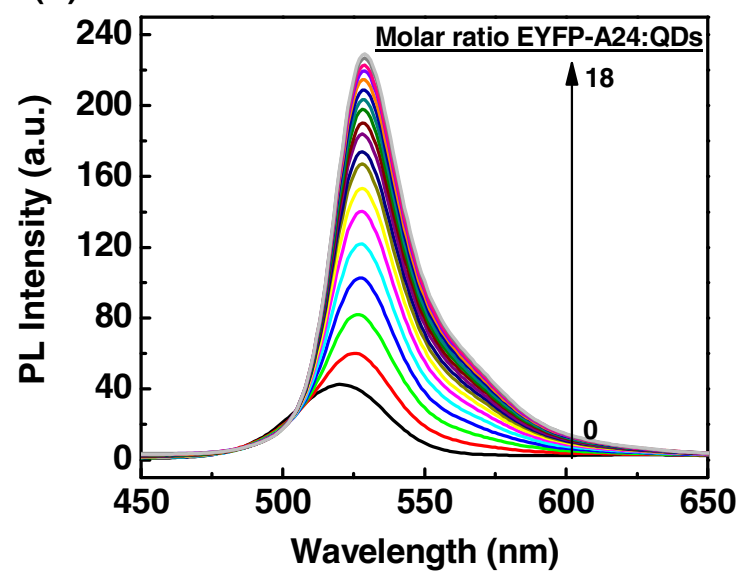

(B)

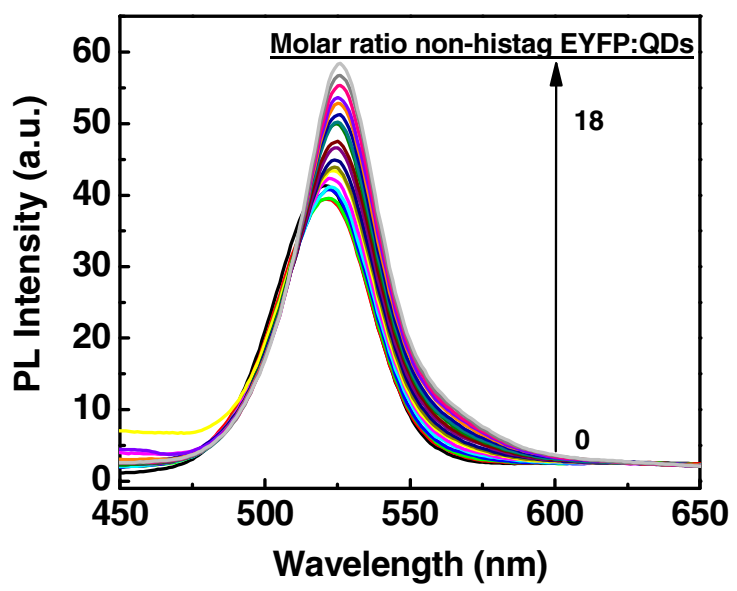

Figure S3. The titration experiments were carried out by gradually adding 0-18 parts of the EYFP-A24 conjugate or non-histidine tagged EYFP to DHLA-QDs solution. (A) PL evolution of EYFP-A24/QD conjugates as the molar ratio increases from 0 to 18 . Each spectrum includes contributions from QD emission, FRET-driven enhancement of EYFP, and EYFP PL due to direct excitation; (B) PL evolution of the solution of 0-18 parts of non-histidine tagged EYFP and one part of DHLA-QDs. The spectra include emission due to directly excited QD and EYFP only. No indications of additional EYFP PL enhancement can be observed.

\section{Simulation of the PL evolution of the QD/EYFP/Atto647 assembly.}

In order to model the temporal evolution of the excitations present in the FRET system, typically a set of rate equations like (1)-(3) representing the changes of the QD (EYFP) (Atto647) excitation as a function of the population and depopulation rates is solved. 


$$
\begin{aligned}
& \frac{d(Q D)}{d t}=Q D \cdot\left(-k_{Q D, \text { rad }}-k_{Q D, \text { nonrad }}-k_{Q D \rightarrow E Y F P} \cdot M_{E Y F P}-k_{Q D \rightarrow \text { Atto } 647} \cdot M_{\text {Atto } 647}\right) \text { (1) } \\
& \frac{d(E Y F P)}{d t}=E Y F P \cdot\left(-k_{E Y F P, \text { rad }}-k_{E Y F P, \text { nonrad }}-k_{E Y F P \rightarrow A t t o 647} \cdot \frac{M_{\text {Atto } 647}}{M_{E Y F P}}+Q D \cdot k_{Q D \rightarrow E Y F P}\right) \text { (2) } \\
& \frac{d(\text { Atto647) }}{d t}=\text { Atto647 } \cdot\left(-k_{\text {Atto647,rad }}-k_{\text {Atto647,nonrad }}+E Y F P \cdot k_{E Y F P \rightarrow A t t o 647} \cdot \frac{M_{E Y F P}}{M_{\text {Atto } 647}}+Q D \cdot k_{Q D \rightarrow A t t o 647}\right) \text { (3) }
\end{aligned}
$$

The resulting functions for QD(t), EYFP (t) and Atto647(t) are then used as fit functions in a least square fit to the data. Since the parameter space spanned by such a set of functions, is rather large, an iterative approach was chosen, which simulates the population of QD, EYFP and Atto647 dye as entries in three 30000 cell long arrays, each cell resembling a time interval of 1 ps.

The initial condition on the arrival of the laser pulse is that all species are excited according to their absorbance at the excitation wavelength used. For all further times, the population of the QD is calculated from the previous time increment by taking into account the radiative and non-radiative decay, the transfer to the EYFP and the transfer to the Atto647 dye. It is important to note that here non-radiative means the intrinsic non-radiative depopulation, which is present even without transfer, due to the non unity quantum yield, as it is visible from the definition of the quantum yield as the ratio of the radiative recombination rate to the sum of radiative and non-radiative recombination rates. A back-filling transfer channel from the EYFP to the QD was initially taken into account in the programming, but found to be negligible in the overall process.

The excited state of the EYFP is populated, apart from the initial laser pulse, by the transfer from the QD donor, while depopulation is governed by radiative and non-radiative rates as well as the transfer transition to the Atto647 dye.

The Atto647 dye itself acts as an acceptor of transferred energy from both the QD and the protein, while depopulation is possible only by non-radiative decay of excitation or photon emission.

The intensities of the PL signals are then calculated by multiplying the population with the 
concentration and the radiative decay rate. Since the signals of the QD and the EYFP overlap, the sum of both contributions is calculated and compared to the measured decay in the corresponding wavelength interval.

Formulae:

Initialization:

QD[0]=pump*AQ, EYFP[0]=pump*AE, Atto[0]=pump*AA.

Calculation for $\mathrm{t}>0$ :

$\mathrm{QD}[\mathrm{i}]=\mathrm{QD}[\mathrm{i}-1] *(1-\mathrm{GQR}-\mathrm{GQNR}-\mathrm{GQE} * \mathrm{ME}-\mathrm{GQA} * \mathrm{MA})+\mathrm{EYFP}[\mathrm{i}-1] * \mathrm{GEQ} * \mathrm{ME}$.

EYFP $[\mathrm{i}]=$ EYFP[i-1]*(1-GER-GENR-GEQ-GEA*MA/ME)+QD[i-1]*GQE

Atto[i] $=$ Atto[i-1]*(1-GAR-GANR)+GEA*EYFP[i-1]*ME/MA+GQA*QD[i-1].

Calculation of PL intensities:

QD_PL[i] $=\mathrm{QD}[\mathrm{i}] * \mathrm{GQR} * \mathrm{MQ}$

EYFP_PL[i] $=\mathrm{EYFP}[\mathrm{i}] * \mathrm{GER} * \mathrm{ME}$

Atto_PL[i]=Atto[i] $*$ GAR $*$ MA

Variable definitions:

pump - intensity of the pump pulse in arbitrary units

AQ - absorbance of the QD at the laser wavelength

$\mathrm{AE}$ - absorbance of the EYFP at the laser wavelength

AA - absorbance of the Atto dye at the laser wavelength

$\mathrm{QD}[\mathrm{i}]$ - the population of one QD unit at time index $\mathrm{i}$

EYFP[i] - the population of one EYFP unit at time index $i$

Atto[i] - the population of one Atto dye unit at time index $i$

GQR - rate of radiative recombination of the QD

GQNR - rate of non-radiative recombination of the QD 
GQE - Rate of QD to EYFP transfer

GQA - rate of QD to Atto dye transfer

GER - rate of radiative recombination of the EYFP

GENR - rate of non-radiative recombination of the EYFP

GEQ - rate of EYFP to QD (back)-transfer

GEA - Rate of EYFP to Atto647 transfer

GAR - rate of radiative recombination of the Atto dye

GANR - rate of non-radiative recombination of the Atto dye

$\mathrm{MQ}$ - concentration of the QD, (set to 1)

ME - concentration of the EYFP, relative to the QD concentration

MA - concentration of the atto dye relative to the QD concentration

\section{Distance dependent FRET of the 3Ch-FRET system 2d-PLE Data}

The full 2D-PLE maps of the $\mathbf{4 a}$ to $\mathbf{4 d}$ systems are shown in Fig. S4. The intensities represented in the color plots enable the identification of wavelength regions suited for further studies. For example the change of emission intensity of the combined donor system QD/EYFP in the presence and the absence of the Atto647 acceptor is found to be almost constant (3\% change) over the range of 350 to $400 \mathrm{~nm}$. This might suggest that the excitons created in the QD relax on a faster time scale than that of the energy transfer and that the particular quantum state in which they are absorbed is of minor importance. This additionally justifies the choice of $372 \mathrm{~nm}$ for the excitation in the PL studies. 

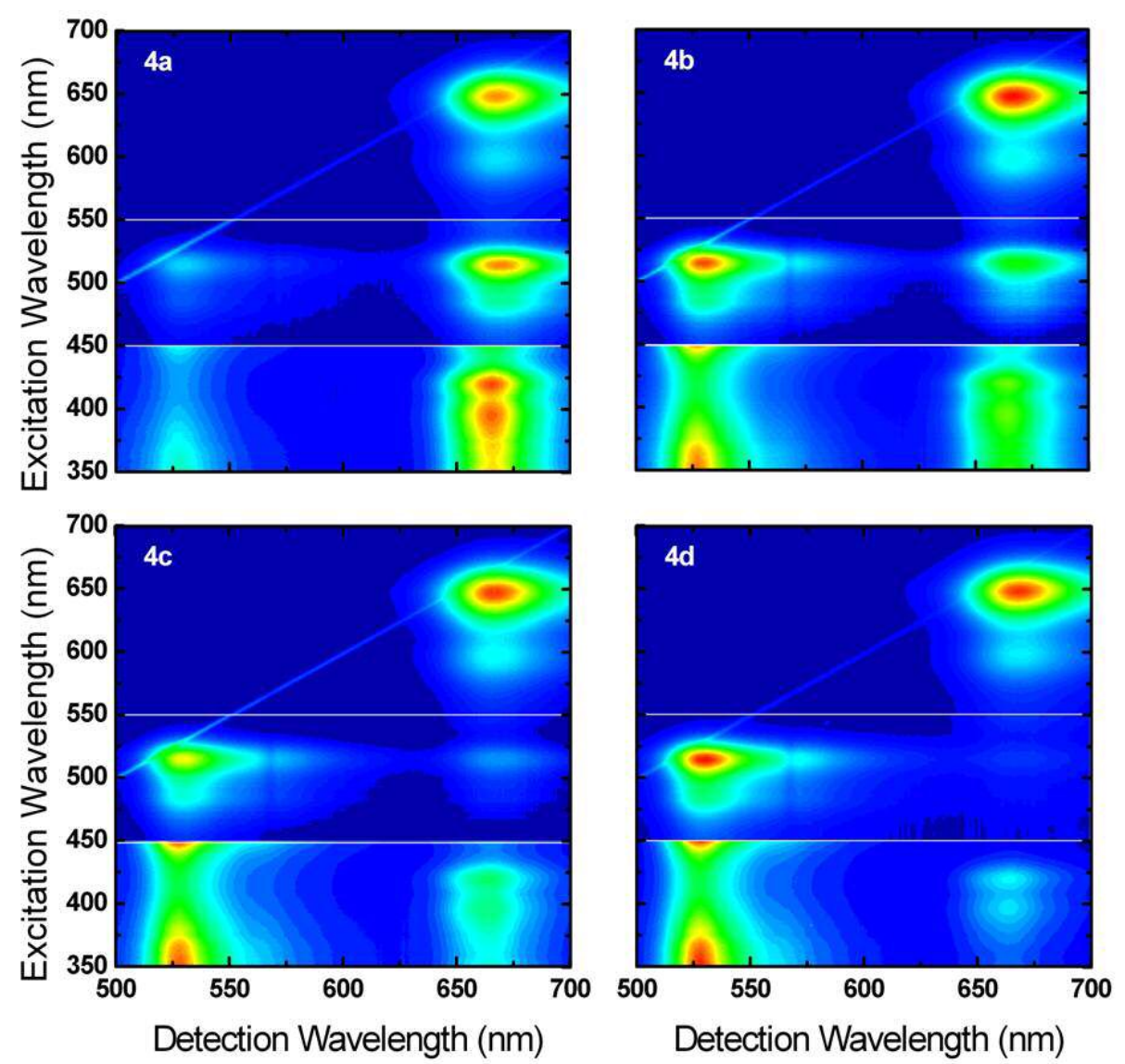

Figure S4. The full excitation-emission characteristic as measured by 2D-PLE for systems $4 \mathbf{4 a - 4 d}$.

Excitation wavelengths longer than $550 \mathrm{~nm}$ show the directly excited Atto647 emission. The 450 to $550 \mathrm{~nm}$ part (enhanced twofold) shows the transfer from the QD/EYFP subsystem to Atto647. The bottom part (enhanced 15x) shows direct Atto647 excitation, QD-Atto647 transfer and the sequential QD-EYFP-Atto647 transfer. 